\title{
REGULAR MODULES
}

\author{
BY \\ J. ZELMANOWITZ
}

\begin{abstract}
In analogy to the elementwise definition of von Neumann regular rings an $R$-module $M$ is called regular if given any element $m \in M$ there exists $f \in \operatorname{Hom}_{R}(M, R)$ with $(m f) m=m$. Other equivalent definitions are possible, and the basic properties of regular modules are developed. These are applied to yield several characterizations of regular self-injective rings. The endomorphism ring $E(M)$ of a regular module ${ }_{R} M$ is examined. It is in general a semiprime ring with a regular center. An immediate consequence of this is the recently observed fact that the endomorphism ring of an ideal of a commutative regular ring is again a commutative regular ring. Certain distinguished subrings of $E(M)$ are also studied. For example, the ideal of $E(M)$ consisting of the endomorphisms with finite-dimensional range is a regular ring, and is simple when the socle of ${ }_{R} M$ is homogeneous. Finally, the self-injectivity of $E(M)$ is shown to depend on the quasi-injectivity of ${ }_{R} M$.
\end{abstract}

Introduction. The notion of (von Neumann) regularity has been extended to modules by D. Fieldhouse [5] and R. Ware [10]. The former author considered arbitrary modules over rings with identity elements while the latter author dealt with projective modules only. Their definitions agree for projectives, and it is in this context that module analogues of ring-theoretic theorems are produced; e.g. [5, Theorem 8.7] and [10, Proposition 2.1 and Theorem 2.12]. In $\S 3$ of [10] we additionally find a study of the endomorphism ring of a regular projective module.

In this paper we will identify a class of modules (which we will at the risk of confusion still call regular) somewhat more restricted than the class of modules introduced in [5], somewhat broader than the regular projectives, but for which structure theorems similar to those mentioned above are still available. Regular modules are introduced in $\S 1$, and their structure examined in $\S 2$. Afterwards, the endomorphism ring of a regular module is studied.

1. Except as indicated, rings will not be assumed to have identity elements, all modules will be left modules, and module homomorphisms will be written on the right. When a ring has an identity element, all modules are assumed to be unitary.

We begin with some basic notation. Given an $R$-module $M$, we set $M^{*}=\operatorname{Hom}_{R}(M, R), \quad E(M)=\operatorname{Hom}_{R}(M, M)$. There are $R$-bilinear functions $():, M \times M^{*} \rightarrow R$ and [, ]: $M^{*} \times M \rightarrow E(M)$ defined by $(m, f)=m f$ and $m[f, n]=(m, f) n$ for all $m, n \in M$ and $f \in M^{*}$. Note that then $[f, m] g=f(m, g)$ for

Received by the editors March 1, 1971.

AMS 1969 subject classifications. Primary 1640, 1650; Secondary 1656.

Key words and phrases. von Neumann regular ring, regular module, finite-dimensional module, projective module, flat module, endomorphism ring, endomorphisms with finitedimensional range, quasi-injective module. 
all $m \in M, f, g \in M^{*}$; and that these functions induce $R-R$-bimodule homomorphisms ( , ): $M \otimes_{E(M)} M^{*} \rightarrow R$ and [ , ]: $M^{*} \otimes_{R} M \rightarrow E(M)$. We let $T(M)$ denote the two-sided ideal of $E(M)$ generated by the image of [ , ].

An $R$-module is called projective if it is a direct summand of a direct sum of copies of $R^{\#}$, where $R^{\#}$ denotes the ring obtained from $R$ by adjoining an identity element in the customary manner when $R$ fails to have an identity element, and $R^{\#}=R$ otherwise. The usual properties of projective modules then hold. If $T(M)$ $=E(M)$, with say $\sum_{i=1}^{t}\left[f_{i}, m_{i}\right]=1 \in E(M), f_{i} \in M^{*}, m_{i} \in M$, then $M$ is projective and $M=\sum_{i=1}^{t} R m_{i}$. Conversely, if $m_{1}, \ldots, m_{t} \in M$ and $N=\sum_{i=1}^{t} R m_{i}$ is projective, then $T(N)=E(N)$. (The usual proof of the "dual basis lemma" establishes these facts. See [1, p. 132].)

Of crucial importance to our discussion is a straightforward variant of a familiar construction. We include the proof for completeness.

LemMA 1.1. Let $M$ be an $R$-module and I a left ideal of $E(M)$. Suppose that $\mu=\mu^{2} \in I, \nu=\nu^{2} \in I$ with $M \mu \cap M \nu=0$ and $\nu \mu=0$. Then there exists $\lambda=\lambda^{2} \in I$ with $M \mu \oplus M \nu=M \lambda$. Consequently $M \mu \oplus M \nu$ is a direct summand of $M$.

Proof. Set $\eta=(1-\mu) \nu$. Then $\eta \in I, \nu \eta=\nu(1-\mu) \nu=\nu^{2}=\nu, \eta^{2}=(1-\mu) \nu \eta=(1-\mu) \nu$ $=\eta, \mu \eta=0=\eta \mu$. Now $M \eta=M(1-\mu) \nu=M \nu$ and $M \nu=M \nu \eta \subseteq M \eta$, so that $M \eta=M \nu$. Since $\mu$ and $\eta$ are orthogonal idempotents $M(\mu+\eta) \subseteq M \mu+M \eta=(M \mu+M \eta)(\mu+\eta)$ $\subseteq M(\mu+\eta)$, so that $M \mu+M \nu=M \mu+M \eta=M(\mu+\eta)$ with $\mu+\eta=(\mu+\eta)^{2} \in I$.

Proposition 1.2. Let $M$ be an R-module such that every cyclic submodule of $M$ is a direct summand of $M$. Then given any direct summand $N$ of $M$ and an element $m \in M$, there exists $m^{\prime} \in M$ such that $N+R^{\#} m=N \oplus R^{\#} m^{\prime}$ and $N+R^{\#} m$ is again a direct summand of $M$.

Proof. Let $M, N, m$ be as above. Since $N$ is a direct summand of $M$, there exists a surjection $\mu: M \rightarrow N$ with $\mu^{2}=\mu$. Now $R^{\#} m=R^{\#} m \mu+R^{\#} m(1-\mu) \subseteq N+R^{\#} m(1-\mu)$, and the latter sum is direct since $N \cap M(1-\mu)=0$. So $N+R^{\#} m \subseteq N+R^{\#} m(1-\mu)$, and since $R^{\#} m(1-\mu) \subseteq R^{\#} m+R^{\#} m \mu \subseteq R^{\#} m+N$ we have $N+R^{\#} m=M \mu \oplus R^{\#} m(1-\mu)$, proving the first statement. Since $R^{\#} m(1-\mu)$ is cyclic, there exists by hypothesis a surjection $\nu: M \rightarrow R^{\#} m(1-\mu)$ with $\nu^{2}=\nu$. So $N+R^{\#} m=M \mu \oplus M \nu$. Now apply the previous lemma.

The reader should note that this proposition remains valid with $R^{\#}$ replaced by $R$, provided that every submodule of the form $R m$ is a direct summand of $R$. An easy induction now establishes the following result.

COROLlARY 1.3. Let $M$ be an R-module which has the property that every cyclic submodule is a direct summand. Then every countably (or finitely) generated submodule of $M$ is a direct sum of cyclic modules and every finitely generated submodule of $M$ is a direct summand of $M$.

We define an $R$-module $M$ to be regular if and only if given any $m \in M$ there exists $f \in M^{*}$ with $m=(m f) m=m[f, m]$. This provides a natural extension of the 
customary elementwise description of a von Neumann regular ring, it being obvious that a regular ring $R$ is regular as a left $R$-module. As an immediate consequence of the definition:

(1.4) A submodule of a regular module is regular.

In particular every left ideal of a regular ring is regular. This provides an ample source of regular modules which are not projective. For example, the ring of linear transformations of a countable dimensional vector space contains nonprojective left ideals. The maximal regular ideal of a ring is clearly a regular module, as is any regular ideal. Some interesting examples of regular projective modules over nonregular rings appear in [10].

Observe that if $m$ is an element of a regular module, then $m \in R m$.

(1.5) A cyclic regular $R$-module is projective and is isomorphic to a left ideal of $R$ generated by an idempotent.

Proof. Let $m$ be a generator of the cyclic regular module $M=R m$. Then from the existence of $f \in M^{*}$ with $m[f, m]=m$ we have that $[f, m]$ is the identity homomorphism on $M$. Hence $T(M)=E(M)$ and $M$ is projective. Furthermore, $f$ is clearly a monomorphism and $M f=R(m f)$ with $(m f)^{2}=(m f)$.

THEOREM 1.6. Let $M$ be a regular module. Then every finitely generated submodule of $M$ is a direct summand of $M$. Every countably (or finitely) generated submodule of $M$ is a direct sum of cyclic regular modules.

Proof. In view of Corollary 1.3 we have only to prove that a cyclic submodule $R m$ of a regular module $M$ is a direct summand. But $m=m[f, m]$ for some $f \in M^{*}$, which implies that $M=R m \oplus \operatorname{ker}[f, m]$.

COROLlaRY 1.7. A countably (or finitely) generated regular module is projective.

We will complete this section with an examination of regular modules which satisfy a chain condition. The upshot of the matter is summarized below.

THEOREM 1.8. Suppose that $M$ is a regular $R$-module which contains no infinite direct sums of submodules. Then $M$ is isomorphic to a finite direct sum of minimal left ideals generated by idempotents.

Proof. Because of Theorem 1.6, every submodule of such a module $M$ must be finitely generated and hence a direct summand of $M$. It follows that $M$ is a direct sum of simple modules $[9$, p. 61$]$, and this sum must of necessity involve only a finite number of nontrivial components. Hence $M=R m_{1} \oplus \cdots \oplus R m_{s}$, with each $R m_{i}$ simple. By (1.5) each $R m_{i}$ is isomorphic to a minimal left ideal of $R$ generated by an idempotent.

COROLLARY 1.9. If $R$ is a commutative ring with identity element then a Noetherian or Artinian regular $R$-module is injective. 
Proof. For such a module satisfies the hypothesis of the previous theorem, hence is a finite direct sum of simple projective modules. But over a commutative ring with identity, a simple module is flat if and only if it is injective [10, Lemma 2.6]; and a finite direct sum of injective modules is injective.

An ideal of a ring is called a regular ideal if it is regular as a subring. Each ring $R$ has a unique largest regular ideal $M(R)$ [7, p. 112]. We call a ring (or module) finite-dimensional if it contains no infinite direct sums of left ideals (or submodules).

Corollary 1.10. If $R$ is a finite-dimensional ring, then $R=M(R) \oplus T$ with $M(R)$ semisimple Artinian and where $T$ is an ideal of $R$ which has no nonzero regular ideals.

Proof. The proof in [7] goes through verbatim, once it is recognized that a finite-dimensional regular ring is semisimple Artinian. This follows from Theorem 1.8 .

We note from the above that when $R$ is a finite-dimensional ring $M(R)$ is in particular a projective $R$-module. The same is now seen to be true for every regular $R$-module.

THEOREM 1.11. If $R$ is either left perfect or is finite-dimensional then every regular $R$-module is projective.

Proof. Recall that a left perfect ring is a ring for which direct limits of projective modules are projective [9, p. 170]. Since a module is a direct limit of its finitely generated submodules, the desired conclusion follows from Corollary 1.7.

Suppose now that $R$ contains no infinite direct sums of left ideals, and let $M$ be a regular $R$-module. Consider $\mathscr{S}=\left\{\sum_{\alpha \in A} \oplus R m_{\alpha} \mid m_{\alpha} \in M\right\}$ partially ordered via $\sum_{\alpha \in A} \oplus R m_{\alpha} \leqq \sum_{\beta \in B} \oplus R m_{\beta}$ if and only if $\left\{m_{\alpha}\right\}_{\alpha \in A} \subseteq\left\{m_{\beta}\right\}_{\beta \in B}$. By Zorn's Lemma there exists $N=\sum_{\alpha \in A} \oplus R m_{\alpha}$ maximal in $\mathscr{S}$. Clearly then $N \cap R m \neq 0$ for every $0 \neq m \in M$. We claim that $N=M$.

Let any $m \in M$ be given. $R m$ is isomorphic to a left ideal of $R$ by (1.5), hence contains no infinite direct sums of submodules. By Theorem 1.8, we can write $R m=R n_{1} \oplus \cdots \oplus R n_{t}$ with each $R n_{i}$ simple. Now $R n_{i} \cap N \neq 0$ for each $i$, so $R n_{i} \cap N$ $=R n_{i} . R m \cap N=\left(R n_{1} \oplus \cdots \oplus R n_{t}\right) \cap N \supseteq \sum_{i=1}^{t} \oplus\left(R n_{i} \cap N\right)=\sum_{i=1}^{t} \oplus R n_{i}=R m$. It follows that $R m \subseteq N$, and so $M=N$ proving that $M$ is projective.

2. We next develop several equivalent characterizations of regularity. Note the following consequence of Theorem 1.6.

(2.1) Over a ring with identity element a regular module is flat.

For a regular module is a direct limit of its finitely generated submodules, each of which is projective.

THEOREM 2.2. For an $R$-module $M$, the following conditions are equivalent:

(1) $M$ is regular.

(2) For every $m \in M, R m$ is projective and is a direct summand of $M$.

(3) For every $m_{1}, \ldots, m_{t} \in M, \sum_{i=1}^{t} R m_{i}$ is projective and is a direct summand of $M$. 
Proof. It is clear from Corollary 1.3 that $(2) \Leftrightarrow(3)$. And (1.5) together with Theorem 1.6 gives $(1) \Rightarrow(2)$. So $(2) \Rightarrow(1)$ remains to be proved.

Assume that $M$ satisfies (2) and let $m$ be an arbitrary element of $M$. By hypothesis $R m$ is projective and $R m \oplus N=M$ for some submodule $N$ of $M$. Since $R m$ is projective there exist elements $f_{i} \in(R m)^{*}$ and $m_{i} \in R m, i=1,2, \ldots, t$, such that $\sum_{i=1}^{t}\left[f_{i}, m_{i}\right] \in E(R m)$ is the identity homomorphism on $R m$. Now $m_{i}=r_{i} m$ for some $r_{1}, r_{2}, \ldots, r_{t} \in R$ and so $\sum_{i=1}^{t}\left[f_{i}, m_{i}\right]=\sum_{i=1}^{t}\left[f_{i} r_{i}, m\right]=\left[\sum_{i=1}^{t} f_{i} r_{i}, m\right]$. Let $g$ denote $\sum_{i=1}^{t} f_{i} r_{i}$ extended across $N$ to $M$. Then $g \in M^{*}$ and $m[g, m]=m$, proving that $M$ is regular.

TheOREM 2.3. Let $R$ be a ring with identity element and $M$ an $R$-module such that every cyclic submodule of $M$ is contained in a projective direct summand of $M$. Then any one of the following conditions is equivalent to $M$ being regular:

(4) Every homomorphic image of $M$ is flat.

(5) $I M \cap N=I N$ for every submodule $N$ of $M$ and every right ideal I of $R$.

(6) For any submodule $N$ of $M$ and any right $R$-module $L$, the natural homomorphism $L \otimes_{R} N \rightarrow L \otimes_{R} M$ is a monomorphism.

In the proof we will utilize a result which is due to Chase [2] for free modules and which was extended in [10] to projectives.

LemMA 2.4. Let $R$ be a ring with identity element. Given a projective $R$-module $M$ and a submodule $K$ of $M, M / K$ is flat if and only if for any $x \in K$ there exists a homomorphism $\alpha_{x}: M \rightarrow K$ with $x \alpha_{x}=x$.

Proof of the theorem. First note that a module for which every cyclic submodule is contained in a projective submodule is a direct limit of projectives, and hence flat.

Assume that $M$ is a regular module and let $K$ be any submodule of $M$. We must prove that $M / K$ is flat, and for this it suffices to prove that every finitely generated submodule of $M / K$ is flat. Such a submodule is of the form $(N+K) / K$ for some finitely generated submodule $N$ of $M$. Since $(N+K) / K \cong N / N \cap K,(N+K) / K$ is a homomorphic image of $N$, a regular projective module by (3) of the preceding theorem and (1.4). Lemma 2.4 now implies that $(N+K) / K$ is flat, completing the proof.

Conversely, let $M$ be as in (4) and take any $m \in M$. By hypothesis $R m \subseteq P$ where $P$ is a projective direct summand of $M$. Composing the natural homomorphisms $M \rightarrow P \rightarrow P / R m$ we see that $P / R m$ is a homomorphic image of $M$ and hence is flat. Applying the lemma to the exact sequence $0 \rightarrow R m \rightarrow P \rightarrow P / R m \rightarrow 0$, we obtain a homomorphism $\alpha: P \rightarrow R m$ with $m \alpha=m$. Thus $R m$ is a direct summand of $P$; and it follows that $R m$ is projective and is a direct summand of $M$. By the previous theorem, $M$ is regular.

In $[9$, p. 133] it is proved that for a flat module $M$ and $N$ a submodule of $M, M / N$ is flat if and only if $I M \cap N=I N$ for every right ideal $I$. And P. M. Cohn [3] has 
shown that for a flat $R$-module $M$ with submodule $N, M / N$ is flat if and only if the natural homomorphism $L \otimes_{R} N \rightarrow L \otimes_{R} M$ is a monomorphism for every right $R$-module $L$. This explains the equivalence of (4), (5) and (6).

In case $M$ is itself a projective module, many of the hypotheses of the previous two theorems become redundant. The equivalence of conditions (2) through (5) for a projective module over a ring with identity forms Proposition 2.1 of [10]. Using Theorem 2.2 the following properties of a regular module are easily established. The proofs involve little modification from those given in [10] for regular projective modules, and will therefore be omitted here.

(2.5) If $M$ is a regular module, then the Jacobson radical of $M$ equals zero.

(2.6) If $M$ is a faithful regular $R$-module, then $R$ is Jacobson-semisimple. Consequently, for any regular $R$-module $M$, the annihilator of $M$ in $R$ is an intersection of maximal left ideals.

(2.7) The singular submodule of a regular module equals zero.

THEOREM 2.8. $\sum_{\alpha \in A} \oplus M_{\alpha}$ is regular if and only if each $M_{\alpha}$ is a regular module.

Proof. Assume that each $M_{\alpha}$ is a regular module. It clearly suffices to show that $M_{1} \oplus M_{2}$ is regular whenever $M_{1}$ and $M_{2}$ are. In fact, given $m_{1} \in M_{1}$ and $m_{2} \in M_{2}$ it suffices by Theorem 2.2 to prove $R\left(m_{1}+m_{2}\right)$ is then projective and a direct summand of $M_{0}=R m_{1} \oplus R m_{2}$.

For $i=1,2$, let $\pi_{i}: M_{0} \rightarrow R m_{i}$ denote the canonical projection homomorphism, and set $\pi=\left.\pi_{1}\right|_{R\left(m_{1}+m_{2}\right)}$. There is an exact sequence

$$
0 \longrightarrow l\left(m_{1}\right) m_{2} \rightarrow R\left(m_{1}+m_{2}\right) \stackrel{\pi}{\rightarrow} R m_{1} \longrightarrow 0
$$

where $l\left(m_{1}\right)=\left\{r \in R \mid r m_{1}=0\right\}$ and $l\left(m_{1}\right) m_{2}=l\left(m_{1}\right)\left(m_{1}+m_{2}\right)$ is the kernel of $\pi$. Since $R m_{1}$ is projective there exists a homomorphism $\mu: R m_{1} \rightarrow R\left(m_{1}+m_{2}\right)$ with $\mu \pi=1 \in E\left(R m_{1}\right)$ and $R\left(m_{1}+m_{2}\right)=R m_{1} \mu \oplus l\left(m_{1}\right) m_{2}$.

Next, there is an exact sequence

$$
0 \rightarrow l\left(m_{1}\right) \rightarrow R \rightarrow R m_{1} \rightarrow 0,
$$

the homomorphism $R \rightarrow R m_{1}$ being defined via $r \rightarrow r m_{1}$. And this sequence is clearly split by any $f \in\left(R m_{1}\right)^{*}$ with $m_{1}=m_{1}\left[f, m_{1}\right]$, so that $R=l\left(m_{1}\right) \oplus R m_{1} f$. Since $m_{1} f=\left(\dot{m}_{1} f\right)^{2}$ it follows that $l\left(m_{1}\right)=R\left(1-\left(m_{1} f\right)\right)$, and hence that $l\left(m_{1}\right) m_{2}$ is a cyclic submodule of $R m_{2}$. This already proves that $R\left(m_{1}+m_{2}\right)=R m_{1} \mu \oplus l\left(m_{1}\right) m_{2}$ $\cong R m_{1} \oplus l\left(m_{1}\right) m_{2}$ is projective.

Finally, $l\left(m_{1}\right) m_{2}$ is a direct summand of $R m_{2}$, so there exists a surjection $\nu: R m_{2} \rightarrow l\left(m_{1}\right) m_{2}$ with $\nu^{2}=\nu$. Then

$$
R\left(m_{1}+m_{2}\right)=R m_{1} \mu \oplus R m_{2} \nu=M_{0}\left(\pi_{1} \mu\right) \oplus M_{0}\left(\pi_{2} \nu\right) .
$$

Since $\left(\pi_{1} \mu\right)^{2}=\pi_{1} \mu,\left(\pi_{2} \nu\right)^{2}=\pi_{2} \nu$ and $\left(\pi_{2} \nu\right)\left(\pi_{1} \mu\right)=0, R\left(m_{1}+m_{2}\right)$ is by Lemma 1.1 a direct summand of $M_{0}$. 
We remark that as a consequence of this theorem every submodule of a direct sum of copies of a regular ring is a regular module.

One might next be tempted to suppose that a (complete) direct product of regular modules is again regular. This is, however, not the case in general. In determining the regular rings which have this property, we obtain new characterizations of regular self-injective rings.

LEMMA 2.9. Suppose that $R$ is a left self-injective ring and $M$ is a cyclic $R$-module with singular submodule equal to zero. Then $M$ is isomorphic to a left ideal of $R$.

Proof. Let $m$ be a generator for $M . l(m)=\{r \in R \mid r m=0\}$ is not an essential left ideal, so $J \cap l(m)=0$ for some nonzero left ideal $J$, and we can assume that $J$ is maximal with respect to this property (so that $J+l(m)$ is an essential left ideal of $R$ ). $J m$ is then an essential submodule of $M$. (For given an arbitrary element $a m \neq 0$ of $M, a \in R^{\#}$, there exists an essential left ideal $I$ with $I a \subseteq J+l(m) . I a \nsubseteq l(m)$ since $a m \neq 0$, so there exist elements $r \in I, 0 \neq s \in J, t \in l(m)$ with $r a=s+t$. But then $0 \neq$ ram $=s m \in J m$.)

Since $J m \cong J$ under the homomorphism $r m \rightarrow r, r \in J$, we can apply the selfinjectivity of $R$ to extend this to a homomorphism $f: M \rightarrow R . f$ is 1-1 since $\operatorname{ker} f \cap J m=0$ and $J m$ is essential in $M$. Thus $f$ is an isomorphism of $M$ onto a left ideal of $R$.

An $R$-module $M$ is defined to be torsion free if for every element $m \in M$, there exists $a \in R^{\#}$ with $\operatorname{Hom}_{R}\left(R^{\#} a m, R\right) \neq 0$. A torsionless module is a submodule of a direct product of copies of $R$; equivalently, $M$ is torsionless if given $0 \neq m \in M$ there exists $f \in M^{*}$ with $m f \neq 0$. A torsionless module is clearly torsion free, as is any submodule of a torsion free module.

THEOREM 2.10. For a regular ring $R$ with identity element the following conditions are equivalent:

(1) $R$ is left self-injective.

(2) Every finitely generated torsion free left $R$-module is projective.

(3) Every torsion free left $R$-module is regular.

(4) Every torsionless left $R$-module is regular, and the maximal left quotient ring of $R$ is a right essential extension of $R$.

(5) $A$ direct product of regular modules is regular, and the maximal left quotient ring of $R$ is a right essential extension of $R$.

When $R$ satisfies any one, and hence all of the above conditions, every finitely generated torsion free $R$-module is isomorphic to a direct sum of principal left ideals of $R$.

Proof. We will proceed by showing that (1) $\Rightarrow(4) \Rightarrow(2) \Rightarrow(1) \Rightarrow(3) \Rightarrow(2)$ and that $(4) \Leftrightarrow(5)$. 
(4) $\Leftrightarrow(5)$. A regular module is torsionless, and a product of torsionless modules is torsionless. So (4) implies (5). Conversely, assume (5) holds. As $R$ is a regular $R$-module, so is every submodule of a direct product of copies of $R$. Thus (5) implies (4).

(1) $\Rightarrow(3)$. Let $M$ be a torsion free $R$-module where $R$ is left self-injective. Suppose that $m$ is an element of the singular submodule of $M$. If $m \neq 0$ then there exists $a \in R$ and $f \in \operatorname{Hom}_{R}(\operatorname{Ram}, R)$ with $(a m) f \neq 0$. On the other hand $(a m) f$ is an element of the singular ideal of $R$ which equals zero (this well-known property of regular rings also follows from (2.7)). Thus the singular submodule of $M$ equals zero. Given any $m \in M$, Lemma 2.9 now implies that $R m$ is isomorphic to a principal left ideal of $R$. Hence $R m$ is injective and regular. So there exists $f \in(R m)^{*}$ with ( $m f) m=m$, and as $R m$ is a direct summand of $M, f$ can be extended to an element of $M^{*}$. This proves that $M$ is regular.

$(1) \Rightarrow(4)$. Assume (1). Since a torsionless module is torsion free, the first statement of (4) follows from the previous paragraph. The second statement of (4) is evident since $R$ is its own maximal left quotient ring.

(3) $\Rightarrow$ (2) follows from Theorem 2.2.

$(4) \Rightarrow(2)$. It suffices to prove that under the hypotheses of (4) any finitely generated torsion free module $M$ is torsionless. Let $Q$ be the maximal left quotient ring of $R$. Given any nonzero element $m \in M$, there exists by hypothesis an element $a \in R$ and a nonzero homomorphism $f \in \operatorname{Hom}_{R}(\operatorname{Ram}, R)$. Since $Q$ is the left injective hull of $R, f$ can be extended to $f^{\prime} \in \operatorname{Hom}_{R}(M, Q)$. Let $m_{1}, m_{2}, \ldots, m_{t}$ generate $M$ over $R$. Since $Q$ is a right essential extension of $R$, there exists $b \in R$ with $\left(m_{1} f^{\prime}\right) b,\left(m_{2} f^{\prime}\right) b, \ldots,\left(m_{t} f^{\prime}\right) b,\left(m f^{\prime}\right) b \in R$ and with $\left(m f^{\prime}\right) b \neq 0$. Then $f^{\prime} b \in M^{*}$ and $m\left(f^{\prime} b\right) \neq 0$, proving that $M$ is torsionless.

(2) $\Rightarrow(1)$. Let $Q$ be the maximal left quotient ring of $R$. Then $Q$ is the left injective hull of $R$. For any $q \in Q, R q+R 1$ is finitely generated and torsion free. (For given any $0 \neq x \in R q+R 1, \operatorname{Hom}_{R}(R a x, R) \neq 0$ for any $a \in R$ with $0 \neq a x \in R$.) $R q+R 1$ is projective, and consequently regular. So there exists $f \in \operatorname{Hom}_{R}(R q+R 1, R)$ with $(1 f) 1=1$, and then $\left.f\right|_{R 1}$ is an isomorphism of $R$ onto $R$. Since $\operatorname{ker} f \cap R=0$, $\operatorname{ker} f=0$. Hence $q f=r f$ for some $r \in R$, and so $q=r \in R$. It follows that $Q=R$ proving that $R$ is left self-injective.

The final statement of the theorem follows from (2) together with Theorem 1.6.

REMARKS. (i) Reviewing the proof of (2) $\Rightarrow(1)$ it becomes clear that one can replace (2) by the equivalent condition:

( $\left.2^{\prime}\right)$ For every pair $q_{1}, q_{2}$ of elements of the maximal left quotient ring of $R$, $R q_{1}+R q_{2}$ is projective.

(ii) The equivalence of (1) and (2) was suggested by R. S. Pierce $\left({ }^{1}\right)$, who proved

( $\left.{ }^{1}\right)$ R. S. Pierce, Modules over commutative regular rings, Mem. Amer. Math. Soc. No. 70 (1967), p. 108. 
it for commutative rings with identity elements. Note that we require an identity element in $R$ only to show that $(2) \Rightarrow(1)\left({ }^{2}\right)$.

(iii) A careful examination of the proof reveals that the regularity of $R$ was only used for about half the implications, the remaining implications being valid in a more general setting.

(iv) We do not know if the second hypothesis in (4) and (5) is in general redun$\operatorname{dant}\left({ }^{3}\right)$. When $R$ is commutative it is redundant, since the maximal left quotient ring of $R$ is then easily seen to be commutative. Consequently, for any commutative regular ring $R$ which is not self-injective there exists a direct product of copies of $R$ which is not a regular $R$-module. (Can this product always be chosen with a countable index set?)

3. The endomorphism ring of a regular module need not be regular. Indeed, Cukerman [4] and Ware [10] have noted that the endomorphism ring of an infinitely generated free module over a regular ring $R$ is regular if and only if $R$ is Artinian. On the other hand, Wiegand [11] has observed that the endomorphism ring of an ideal of a commutative regular ring is again a commutative regular ring. This is not true for noncommutative rings, as is seen in the next example.

EXAMPLE 3.1. A left ideal of a regular ring whose endomorphism ring is not regular.

Let $R$ be a ring of column-finite countable matrices over a field. Let $\left\{e_{i j} \mid 1 \leqq i, j<\infty\right\}$ denote the usual matrix units of $R$. Set

$$
J=\prod_{j \text { odd }} R e_{1 j} \oplus \sum_{j \text { even }} \oplus R e_{1 j},
$$

a left ideal of $R$. Define $\alpha: J \rightarrow J$ by setting $\left(\prod_{j \text { odd }} R e_{1 j}\right) \alpha=0$ and $e_{1 j} \alpha=e_{1(j-1)} \in J$ for $j$ even. Then $\alpha$ extends linearly to an $R$-endomorphism of $J$ and $J \alpha=\sum_{j \text { odd }} \oplus R e_{1 j}$. Were $E(J)$ regular, $J_{\alpha}$ would be a direct summand of $J$ and hence of $\prod_{j \text { odd }} R e_{1 j}$. But this is easily seen not to be the case: for since $R=\prod_{j \text { odd }} \operatorname{Re}_{1 j} \oplus \prod_{j \text { even }} \operatorname{Re}_{1 j}$, $J \alpha=\sum_{j \text { odd }} \oplus R e_{1 j}$ would then be a principal left ideal, in clear violation of the fact that it is an infinite direct sum.

What then can one say affirmatively about the endomorphism ring of a regular module or ideal. One minor observation:

(3.2) The endomorphism ring of a regular module is a semiprime ring.

For if $0 \neq \alpha \in E(M)$ with ${ }_{R} M$ regular, choose $m \in M$ so that $m \alpha \neq 0$. Since $M$ is regular there exists $f \in M^{*}$ with $m \alpha=m \alpha[f, m \alpha]=m(\alpha[f, m] \alpha)$. It follows that $\alpha[f, m] \alpha \neq 0$, and hence that $E(M)$ is semiprime.

$\left({ }^{2}\right)$ For some information, see V. Cateforis and F. Sandomierski, On modules of singular submodule zero, Canad. J. Math. 23 (1971), 346.

${ }^{(3)}$ The equivalence of (1) and (2) has also been noted by E. Gentile (Purity and algebraic closure, Rev. Un. Mat. Argentina 24 (1968), 37-45), V. Cateforis (On regular self-injective rings, Pacific J. Math 30 (1969), 39-45), and Rangaswamy and Vanaja (A note on modules over regular rings, Bull. Austral. Math. Soc. 4 (1971), 57-62), and no doubt by countless other authors. 
Before we can turn to our next result we need an observation which is essentially contained in [6].

LEMMA 3.3. Let $\alpha \in$ center of $E(M)$. Then there exists an element $\beta \in$ center of $E(M)$ with $\alpha \beta \alpha=\alpha$ if and only if $M=M \alpha \oplus \operatorname{ker} \alpha$.

Proof. Suppose that such a $\beta$ exists. Set $\pi=\beta \alpha=\alpha \beta$. Then $\pi^{2}=\pi ; M \alpha=M \alpha \pi$ $\subseteq M \pi$ and $M \pi=M \beta \alpha \subseteq M \alpha$ so that $M \alpha=M \pi ;$ and $\operatorname{ker} \alpha=\operatorname{ker} \pi$ since $\pi=\alpha \beta$ and $\alpha=\pi \alpha$. Clearly $M=M \pi \oplus M(1-\pi)=M \pi \oplus \operatorname{ker} \pi$, and so $M=M \alpha \oplus \operatorname{ker} \alpha$.

Conversely, suppose that $\alpha \in$ center of $E(M)$ and $M=M \alpha \oplus \operatorname{ker} \alpha$. Given $m \in M$ write $m=n \alpha+k$ with $n \in M$ and $k \in \operatorname{ker} \alpha$; and in turn write $n=n_{1} \alpha+k_{1}$ with $n_{1} \in M$ and $k_{1} \in \operatorname{ker} \alpha$. Then $m \alpha=\left(\left(n_{1} \alpha+k_{1}\right) \alpha+k\right) \alpha=\left(n_{1} \alpha\right) \alpha^{2}$. Set $x_{m}=n_{1} \alpha \in M \alpha$. Observe that $x_{m}$ is the unique element of $M \alpha$ such that $m \alpha=x_{m} \alpha^{2}$; for if $y \in M \alpha$ with $m \alpha=y \alpha^{2}$, then $\left(y-x_{m}\right) \alpha^{2}=0$ so that $\left(y-x_{m}\right) \alpha \in M \alpha \cap \operatorname{ker} \alpha=0$, and then also $y-x_{m} \in M \alpha \cap \operatorname{ker} \alpha=0$ so that $y=x_{m}$. It is easy to check that $x_{r m}=r x_{m}$, $x_{m+n}=x_{m}+x_{n}, x_{m \gamma}=x_{m} \gamma$ for any $m, n \in M, r \in R, \gamma \in E(M)$. Consequently there is a homomorphism $\beta \in E(M)$ defined by $m \beta=x_{m}$. For any $m \in M, m \alpha \beta \alpha=(m \alpha \beta) \alpha$ $=x_{m \alpha} \alpha=x_{m} \alpha^{2}=m \alpha$, so that $\alpha \beta \alpha=\alpha$. It remains only to show that $\beta$ is in the center of $E(M)$. But this is easy, for given any $\gamma \in E(M)$ and any $m \in M, m \gamma \beta=x_{m \gamma}=x_{m} \gamma$ $=m \beta \gamma$.

THEOREM 3.4. Suppose that ${ }_{R} M$ is a regular module. Then the center of $E(M)$ is a regular ring.

Proof. Let $\alpha \in$ center of $E(M)$. Given any $m \in M$ choose $f \in M^{*}$ with $m \alpha$ $=m \alpha[f, m \alpha]=m[f, m] \alpha^{2}$. Then $m=m[f, m] \alpha+(m-m[f, m] \alpha)$ with $m[f, m] \alpha \in M \alpha$ and $m-m[f, m] \alpha \in \operatorname{ker} \alpha$. So $M=M \alpha+\operatorname{ker} \alpha$. If $m \alpha \in M \alpha \cap \operatorname{ker} \alpha$, then from the above equation it follows that $m \alpha=m[f, m] \alpha^{2}=(m, f)\left(m \alpha^{2}\right)=0$. Hence $M \alpha \cap \operatorname{ker} \alpha$ $=0$ and $M=M \alpha \oplus$ ker $\alpha$. Now apply the previous lemma.

COROLlaRY 3.5. Suppose that $J$ is a regular left ideal of $R$. If $J$ is commutative then $E(J)$ is a commutative regular ring.

Proof. First recall that a regular (left) ideal is a (left) ideal which is itself a regular ring. (For example, any ideal of a regular ring is a regular ideal.) A regular left ideal $J$ of $R$ is in particular a regular left $R$-module. To complete the proof it suffices to show that $E(J)$ is commutative when $J$ is. Let $\alpha, \beta \in E(J)$ and let $x$ be any element of $J$. Choose $y \in J$ with $x y x=x$. Then

$$
\begin{aligned}
x \alpha \beta & =(x y x) \alpha \beta=(x y(x \alpha)) \beta=(y(x \alpha) x) \beta=y(x \alpha)(x \beta)=y(x \beta)(x \alpha) \\
& =(y(x \beta) x) \alpha=(x y(x \beta)) \alpha=(x y x) \beta \alpha=x \beta \alpha
\end{aligned}
$$

proving that $\alpha \beta=\beta \alpha$.

N. Funayama has recently observed that Lemma 3.3 can easily be used to prove that the ring of $R-R$-bimodule endomorphisms of a regular ideal of $R$ is a commutative regular ring. 
We have not been able to determine precisely which regular modules have commutative (and hence necessarily regular) endomorphism rings. The next result gives some information about this situation.

THEOREM 3.6. Suppose that ${ }_{R} M$ is a regular module with $E(M)$ commutative. If either $R$ is left self-injective or ${ }_{R} M$ is projective, then $M$ is isomorphic to a left ideal of $R$.

Proof. If ${ }_{R} M$ is a module with $E(M)$ commutative, then $N \alpha \subseteq N$ for every endomorphic image $N$ of $M$ and every $\alpha \in E(M)$. (Let $N=M \beta$ with $\beta \in E(M)$; then $N \alpha=M \beta \alpha=M \alpha \beta \subseteq M \beta=N$.) Consequently, if ${ }_{R} M$ is additionally a regular module, then $N \alpha \subseteq N$ for every submodule $N$ of $M$ and every $\alpha \in E(M)$. (For given any $n \in N$, $R n$ is a direct summand of $M$, so a fortiori is an endomorphic image of $M$. Hence $n \alpha \in R n \alpha \subseteq R n \subseteq N$.)

Now let $N=\sum_{i \in I} \oplus R m_{i}$ be any submodule of $M$ which is a direct sum of cyclic submodules. For each $i \in I$ write $R m_{i}=M \alpha_{i}$ where $\alpha_{i} \in E(M)$, and set $N_{i}$ $=\sum_{j \in I, j \neq i} R m_{j}$. By Lemma 3.3, $M=M \alpha_{i} \oplus \operatorname{ker} \alpha_{i}=R m_{i} \oplus \operatorname{ker} \alpha_{i}$ for each $i \in I$. By the first paragraph, $N_{i} \alpha_{i} \subseteq N_{i} \cap R m_{i}=0$, so that $N_{i} \subseteq \operatorname{ker} \alpha_{i}$ for each $i \in I$. Also $\operatorname{Hom}_{R}\left(R m_{i}, N_{i}\right)=0$ since any $\gamma \in \operatorname{Hom}_{R}\left(R m_{i}, N_{i}\right)$ can be extended across ker $\alpha_{i}$ via $\left(\operatorname{ker} \alpha_{i}\right) \gamma=0$ to an element $\gamma \in E(M)$; and then $\left(R m_{i}\right) \gamma \subseteq R m_{i} \cap N_{i}=0$ so that $\gamma=0$. In particular, $\operatorname{Hom}_{R}\left(R m_{i}, R m_{j}\right)=0$ whenever $i, j \in I$ with $i \neq j$.

For each $i \in I$, there exists by (1.5) an $R$-isomorphism $f_{i}: R m_{i} \rightarrow R e_{i}$ where $m_{i} f_{i}=e_{i}=e_{i}^{2} \in R$ and $\left(m_{i} f_{i}\right) m_{i}=m_{i}$. We can extend $f_{i}$ to an $R$-homomorphism $g_{i} \in M^{*}$ by defining $g_{i}=0$ on $\operatorname{ker} \alpha_{i} . g=\sum_{i \in I}\left(\left.g_{i}\right|_{N}\right)$ is then a well-defined $R$ homomorphism from $N$ to $R$, and $N g=\sum_{i \in I} R m_{i} f_{i}=\sum_{i \in I} R e_{i}$. We claim that $g$ is a monomorphism, and to see this it suffices to prove that $\sum_{i \in I} R e_{i}$ is a direct sum.

Set $L=\sum_{i \in I} R e_{i}, L_{i}=\sum_{j \in I, j \neq i} R e_{j}$, and suppose that $a \in R e_{i} \cap L_{i}$. Since $R e_{i} \cong R m_{i}$ is regular, $R a$ is a direct summand of $R e_{i}$. Hence if $a \neq 0$, then $0 \neq \operatorname{Hom}_{R}\left(R e_{i}, R a\right)$ $\subseteq \operatorname{Hom}_{R}\left(R e_{i}, L_{i}\right)$. Since $L_{i}=\sum_{j \in I, j \neq i} \oplus R e_{j}, \operatorname{Hom}_{R}\left(R e_{i}, L_{i}\right) \neq 0$ implies that there

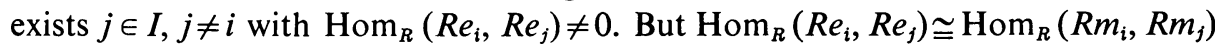
$=0$. Hence $a=0$ and $\sum_{i \in I} R e_{i}$ is a direct sum, establishing the fact that $g$ is a monomorphism of $N$ into $R$.

If ${ }_{R} M$ is projective then we can choose $N=M$ giving the desired conclusion. For a projective module is a direct sum of countably generated projectives (by [8, Theorem 1] applied to a regular projective module), each of which is by Corollary 1.3 a direct sum of cyclic submodules. In general, we may use a Zorn's lemma argument as in the proof of Theorem 1.11 to choose $N$ with the property that $N \cap R m \neq 0$ for every nonzero element $m \in M$. If now ${ }_{R} R$ is injective we can extend $g$ to an $R$-homomorphism $g^{\prime}: M \rightarrow R$. Since $\operatorname{ker} g^{\prime} \cap N=\operatorname{ker} g=0$, it follows that $\operatorname{ker} g^{\prime}=0$ and $M$ is therefore isomorphic to a left ideal of $R$.

One final comment on a related result is in order. It follows directly from Corollary 4.3 of [12] together with Theorem 1.8 of this paper that over a prime ring $R$ a regular module with a commutative endomorphism ring is isomorphic to a minimal left ideal of $R$. 
(3.7) Let $R$ be a commutative ring and $P$ a projective $R$-module such that $E(P)$ is a regular ring. Then $P$ is a regular module.

For rings with identity elements this is Theorem 3.9 of [10]. We note that with but minor modification the proof given there is valid for rings without identity elements. It can be extended as follows.

THEOREM 3.8. Let $R$ be a commutative ring, and let $M$ be an $R$-module with the property that every cyclic submodule of $M$ is contained in a projective direct summand of $M$. If $E(M)$ is a regular ring then $M$ is a regular module.

Proof. Let $m \in M$ be given. By hypothesis $R^{\#} m \subseteq P$ where $P$ is a projective direct summand of $M$. Letting $\pi$ denote the canonical projection homomorphism of $M$ onto $P, E(P) \cong \pi E(M) \pi$, so that $E(P)$ is a regular ring. By (3.7), $P$ is regular. Hence there exists $g \in P^{*}$ with $(m g) m=m$. Extending $g$ across a complementary summand of $P$ in $M$ to a homomorphism $f \in M^{*}$, we have $(m f) m=m$, proving that $M$ is regular.

4. We have already seen that the center of $E(M)$ is a regular ring when $M$ is a regular $R$-module. In this section it is our intention to investigate other distinguished subsets of $E(M)$. Three come immediately to mind; namely, $T(M)$, $F(M)=\{\alpha \in E(M) \mid M \alpha$ is finitely generated $\}$, and $G(M)=\{\alpha \in E(M) \mid M \alpha$ is finite dimensional\}. $T(M)$ is an ideal of $E(M), F(M)$ is a multiplicative subsemigroup of $E(M)$, and $G(M)$ will shortly be seen to be an ideal of $E(M)$.

THEOREM 4.1. If $M$ is a regular $R$-module then given $\alpha \in F(M)$ there exists $\beta \in F(M)$ with $\alpha=\alpha \beta \alpha$.

Proof. Let $\alpha \in F(M), M$ a regular module. One can write $M \alpha=R m_{1} \alpha \oplus \cdots$ $\oplus R m_{t} \alpha, M=M \alpha \oplus N$ for some elements $m_{1}, \ldots, m_{t} \in M$ and $N$ a submodule of $M$. Since each $R m_{i} \alpha$ is regular, there exist homomorphisms $g_{i} \in\left(R m_{i} \alpha\right)^{*}$ for $i=1, \ldots, t$ such that $\left(m_{i} \alpha g_{i}\right) m_{i} \alpha=m_{i} \alpha$. Set

$$
M_{i}=R m_{1} \alpha \oplus \cdots \oplus R m_{i-1} \alpha \oplus R m_{i+1} \alpha \oplus \cdots \oplus R m_{t} \alpha \oplus N ;
$$

$R m_{i} \alpha \oplus M_{i}=M$ for each $i=1, \ldots, t$. Let $f_{i} \in M^{*}$ denote $g_{i}$ extended across $M_{i}$ to $M$ via $\left(M_{i}\right) f_{i}=0$; and set $\beta=\sum_{i=1}^{t}\left[f_{i}, m_{i}\right] \in T(M)$. Then $\alpha \beta \alpha=\alpha$; for, given any $m \in M$, write $m \alpha=\sum_{i=1}^{t} r_{i} m_{i} \alpha$ with $r_{1}, \ldots, r_{t} \in R$, and then

$$
\begin{aligned}
m \alpha \beta \alpha & =\left(\sum_{i=1}^{t} r_{i} m_{i} \alpha\right)\left(\sum_{j=1}^{t}\left[f_{j}, m_{j} \alpha\right]\right)=\sum_{i, j=1}^{t} r_{i}\left(m_{i} \alpha, f_{j}\right) m_{j} \alpha \\
& =\sum_{i=1}^{t} r_{i}\left(m_{i} \alpha g_{i}\right) m_{i} \alpha=\sum_{i=1}^{t} r_{i} m_{i} \alpha=m \alpha .
\end{aligned}
$$

Finally observe that $\beta \in F(M)$ since

is finitely generated.

$$
M \beta=\left(R m_{1} \alpha \oplus \cdots \oplus R m_{t} \alpha \oplus N\right) \beta=\sum_{i=1}^{t} R\left(m_{i} \alpha g_{i}\right) m_{i}
$$


Corollary 4.2 [10, Theorem 3.6]. If $M$ is a finitely generated regular module, then $E(M)$ is a regular ring.

THEOREM 4.3. $G(M)$ is a regular ideal of $E(M)$ and $G(M) \subseteq F(M) \subseteq T(M)$.

Proof. For any $\alpha \in G(M), M \alpha$ is by Theorem 1.8 a finite direct sum of simple modules. Hence $G(M) \subseteq F(M)$. For any $\alpha, \beta \in G(M)$ and $\gamma \in E(M), M(\alpha+\beta)$ $\subseteq M \alpha+M \beta, M \gamma \alpha \subseteq M \alpha$, and $M \alpha \gamma=(M \alpha) \gamma$. Since finite sums, submodules and homomorphic images of a finite direct sum of simple modules are again of the same type, it follows that $\alpha+\beta, \gamma \alpha, \alpha \gamma \in G(M)$ proving that $G(M)$ is an ideal of $E(M)$.

To see that $G(M)$ is regular, simply use Theorem 4.1 to choose for a given $\alpha \in G(M)$ an element $\beta \in F(M)$ with $\alpha=\alpha \beta \alpha$. Then $\alpha=\alpha(\beta \alpha \beta) \alpha$ with $\beta \alpha \beta \in G(M)$ since $G(M)$ is an ideal of $E(M)$. It remains to prove that $F(M) \subseteq T(M)$. Let $\alpha \in F(M)$ and choose $\beta \in T(M)$ as in the proof of Theorem 4.1 with $\alpha=\alpha \beta \alpha$. Since $T(M)$ is an ideal of $E(M), \alpha=\alpha \beta \alpha \in T(M)$.

COROllaRY 4.4. If $R$ is finite dimensional and $M$ is a regular $R$-module then $G(M)=F(M)=T(M)$.

Proof. First note that every finitely generated submodule $N$ of $M$ is finite dimensional. For by Theorem 1.6, $N$ is isomorphic to a finite direct sum of left ideals of $R$, each of which is finite dimensional. In particular, $F(M) \subseteq G(M)$.

Next let $\alpha=\sum_{i=1}^{t}\left[f_{i}, m_{i}\right] \in T(M), f_{i} \in M^{*}, m_{i} \in M$. Then $M \alpha \subseteq \sum_{i=1}^{t}\left(M f_{i}\right) m_{i}$ $\subseteq \sum_{i=1}^{t} R m_{i}$. Since $\sum_{i=1}^{t} R m_{i}$ is finite dimensional, so is $M \alpha$. Hence $T(M) \subseteq G(M)$. Now apply the preceding theorem.

In analogy to the situation for the ring of linear transformations of a vector space, we will show that $G(M)$ is a simple ring when the simple submodules of $M$ are all of the same isomorphism type (such a module is said to have a homogeneous socle). The proof for vector spaces is easily adapted, the key step being the following lemma, whose proof is sketched below for the sake of completeness.

Lemma 4.5. Assume that $M$ is an $R$-module with the property that every finite direct sum of simple submodules of $M$ is a direct summand of $M$. Suppose that $\mu=\mu^{2} \in G=G(M)$ and that $N$ is a submodule of $M$ with both $M \mu$ and $N$ finite direct sums of simple modules of the same isomorphism type. Then there exists $\nu=\nu^{2} \in G \mu G$ with $M \nu=N$.

Proof. Write $M \mu=M_{1} \oplus \cdots \oplus M_{t}$ with each $M_{i}$ simple. First assume $N$ is simple. Choose an isomorphism $\alpha: N \rightarrow M_{1}$, and extend $\alpha$ across a complementary summand of $N$ in $M$, so that $\alpha \in G(M)$. Define $\beta: M \mu \rightarrow N$ via $\left.\beta\right|_{M_{1}}=\bar{\alpha}^{1},\left.\beta\right|_{M_{i}}=0$ $(i=2, \ldots, t)$, and extend $\beta$ across a complementary summand of $M \mu$ in $M$, so that $\beta \in G(M)$. Set $\nu=\alpha \mu \beta \in G \mu G$. Then $M \nu=N$ and $\left.\nu\right|_{N}=1$ so that $\nu^{2}=\nu$.

Suppose now $N=N_{1} \oplus \cdots \oplus N_{t}$ with each $N_{i}$ simple. By induction, there exists $\nu^{\prime}=\left(\nu^{\prime}\right)^{2} \in G \mu G$ with $M \nu^{\prime}=N_{1} \oplus \cdots \oplus N_{t-1}=N^{\prime}$. Write $N_{t}=R n_{t}$ and say $n_{t} \nu^{\prime}$ 
$=\sum_{i=1}^{t-1} n_{i}, n_{i} \in N_{i}$. Then clearly $n=n_{t}-\sum_{i=1}^{t-1} n_{i} \in \operatorname{ker} \nu^{\prime}$ and $N=N^{\prime} \oplus R n$, so that $R n$ is simple. By the first paragraph, there exists $\nu^{\prime \prime}=\left(\nu^{\prime \prime}\right)^{2} \in G \mu G$ with $M \nu^{\prime \prime}=R n$. Set $\nu=\nu^{\prime}+\nu^{\prime \prime}-\nu^{\prime} \nu^{\prime \prime} \in G \mu G$. For any $n^{\prime} \in N^{\prime}, n^{\prime} \nu=n^{\prime}+n^{\prime} \nu^{\prime \prime}-n^{\prime} \nu^{\prime \prime}=n^{\prime}$, while $n \nu=n \nu^{\prime \prime}$ $=n$, so that $\left.\nu\right|_{N}=1$. Hence $M \nu \supseteq N$. On the other hand $M \nu \subseteq M \nu^{\prime}+M \nu^{\prime \prime} \subseteq N$, so that $M \nu=N$ and $\nu^{2}=\nu$.

THEOREM 4.6. If $M$ is a regular $R$-module with homogeneous socle then $G(M)$ is a simple ring.

Proof. Let $0 \neq \alpha \in G=G(M)$; we must show that $G \alpha G=G$. Since $G(M)$ is regular we can choose $\beta \in G(M)$ with $\alpha=\alpha \beta \alpha$. Then $(\beta \alpha)^{2}=\beta \alpha \in G(M)$. Given any $\gamma \in G(M)$ we apply the lemma to choose $\nu=\nu^{2} \in G(\beta \alpha) G$ with $M \nu=M \gamma$. Then $\gamma=\gamma \nu \in \gamma G(\beta \alpha) G \subseteq G \alpha G$, proving that $G \alpha G=G$.

For $M$ an arbitrary regular module, the situation is not much more complicated. $G(M)$ is then the direct sum of ideals $G_{i}(M)$, one for each homogeneous component $L_{i}$ of the socle of $M$. Each $G_{i}(M)$ is simple, and in fact

$$
G_{i}(M)=G(M) \cap \operatorname{Hom}_{R}\left(M, L_{i}\right) .
$$

The details are elementary.

COROLlary 4.7. If $R$ is a simple Artinian ring and $M$ is any $R$-module then $G(M)$ is a simple regular ring.

We conclude this section with a description of the regular modules with semisimple endomorphism rings.

THEOREM 4.8. Let $M$ be a regular module. Then $E(M)$ is semisimple with minimum condition if and only if ${ }_{R} M$ is finite dimensional.

Proof. If $M$ contains no infinite direct sums, then by Theorem $1.8 M$ is a direct sum of finitely many simple modules and so has a semisimple endomorphism ring. Conversely, suppose that $E(M)$ is semisimple with $M$ a regular module, and that $\sum_{i \in I} \oplus M_{i} \subseteq M$ for some collection of nonzero submodules $M_{i}$ of $M$. It is easy to see that $\sum_{i \in I}\left[M^{*}, M_{i}\right]$ then forms a direct sum of nonzero left ideals of $E(M)$. So $I$ must be a finite index set.

5. In this brief concluding section we determine exactly when the endomorphism ring of a regular module is left self-injective. The next result is of some independent interest.

THEOREM 5.1. Assume that $M$ is an R-module with the property that for every cyclic submodule $L$ of $M$ there exists a surjection of $M$ onto $L$. If $E(M)$ is left selfinjective, then ${ }_{R} M$ is quasi-injective.

Proof. Let $N$ be an $R$-submodule of $M$ and let $\alpha \in \operatorname{Hom}_{R}(N, M)$. We must prove that $\alpha$ can be extended to an element of $E=E(M)$. Set $I=\operatorname{Hom}_{R}(M, N)$, a left ideal of $E$. Define $\theta: I \rightarrow E$ via $(\gamma) \theta=\gamma \circ \alpha, \gamma \in I$. Clearly $\theta \in \operatorname{Hom}_{E}(I, E)$. Since 
${ }_{E} E$ is injective and contains an identity element, there exists a homomorphism $\alpha^{\prime} \in E$ with $(\gamma) \theta=\gamma \circ \alpha^{\prime}$ for all $\gamma \in I$. The proof is concluded by demonstrating that $\left.\alpha^{\prime}\right|_{N}=\alpha$.

For any $n \in N$ there exists by hypothesis a surjection $\beta \in \operatorname{Hom}_{R}\left(M, R^{\#} n\right)$. Choose $m \in M$ with $m \beta=n$. Note that $\beta \in I$. Then $n \alpha^{\prime}=(m \beta) \alpha^{\prime}=m\left(\beta \circ \alpha^{\prime}\right)=m(\beta \circ \alpha)=(m \beta) \alpha$ $=n \alpha$, so that $\left.\alpha^{\prime}\right|_{N}=\alpha$.

THEOREM 5.2. For $M$ a regular $R$-module, $E(M)$ is left self-injective if and only if ${ }_{R} M$ is quasi-injective; and when this is the case $E(M)$ is regular.

Proof. The "only if" part is a consequence of the previous result, it being evident that a regular module satisfies the hypothesis of Theorem 5.1. While it is possible to give an independent proof of the remainder of this theorem, it is actually a special case of a more general result. It is known that the endomorphism ring $E$ of an injective or quasi-injective module ${ }_{R} M$ is regular and left self-injective when $J(E)$, the Jacobson radical of $E$, equals zero. Furthermore, $J(E)=\{\alpha \in E \mid \operatorname{ker} \alpha \cap N$ $\neq 0$ for every $R$-submodule $N \neq 0$ of $M\}$. (See for example [9,pp. 102-104].) When $M$ is a regular $R$-module it is a simple matter using (2.7) to verify that $J(E)=0$.

\section{REFERENCES}

1. H. Cartan and S. Eilenberg, Homological algebra, Princeton Univ. Press, Princeton, N. J., 1956. MR 17, 1040.

2. S. U. Chase, Direct products of modules, Trans. Amer. Math. Soc. 97 (1960), 457-473. MR 22 \#11017.

3. P. M. Cohn, On the free product of associative rings. I, Math. Z. 71 (1959), 380-398. MR 21 \#5648.

4. G. M. Cukerman, Rings of endomorphisms of free modules, Sibirsk. Mat. Ž. 7 (1966), 1161-1167. (Russian) MR 34 \#2631.

5. D. J. Fieldhouse, Pure theories, Math. Ann. 184 (1969), 1-18. MR 40 \#5699.

6. N. Funayama, Imbedding a regular ring with identity, Nagoya Math. J. 27 (1966), 61-64. MR 33 \#4081.

7. I. Kaplansky, Fields and rings, Univ. of Chicago Press, Chicago, Ill., 1969.

8. - - Projective modules, Ann. of Math. (2) 68 (1958), 372-377. MR 20 \#6453.

9. J. Lambek, Lectures on rings and modules, Blaisdell, Waltham, Mass., 1966. MR 34 \#5857.

10. R. Ware, Endomorphism rings of projective modules, Trans. Amer. Math. Soc. 155 (1971), 233-259.

11. R. Wiegand, Endomorphism rings of ideals in a commutative regular ring, Proc. Amer. Math. Soc. 23 (1969), 442-449. MR 40 \#7245.

12. J. Zelmanowitz, Commutative endomorphism rings, Canad. J. Math. 23 (1971), 69-76. 